\title{
Avaliação muscular subjetiva como parâmetro complementar de diagnóstico nutricional em pacientes no pré-operatório
}

\author{
Subjective muscle assessment as a \\ complementary parameter for the nutritional \\ diagnosis in presurgical patients
}

Ionar Figueredo Bonfim REZENDE

Andréa da Silva ARAÚJO2

Martha França SANTOS²

Lílian Ramos SAMPAIO ${ }^{3}$

Rosângela Passos de Jesus MAZZA

\section{RE S U M O}

\section{Objetivo}

Comparar a Avaliação Muscular Subjetiva com métodos objetivos de avaliação nutricional em pacientes que serão submetidos à cirurgia.

\section{Métodos}

Trata-se de um estudo de comparação entre métodos de avaliação nutricional, com características transversais, envolvendo dados primários em pacientes no pré-operatório. Realizaram-se medidas antropométricas, laboratoriais e a Avaliação Muscular Subjetiva para diagnóstico nutricional dos pacientes. Utilizou-se o Coeficiente de Tau-b de Kendall, adotando-se como concordância excelente entre os métodos valores maiores ou iguais a $80 \%$.

\section{Resultados}

Foram incluídos no protocolo 168 pacientes adultos, de ambos os sexos, com indicação de cirurgia de médio e grande porte. Para a Avaliação Muscular Subjetiva os valores observados no teste de concordância inter e intra-examinador foram $77,0 \%$ e $89,0 \%$, respectivamente. Os grupamentos musculares que apresentaram maior percentual de alterações tróficas foram o temporal e masseter para ambos os sexos (feminino=53,5\%; masculino=82,0\%). Para o sexo feminino, o grau de concordância entre a Avaliação Muscular Subjetiva e os

\footnotetext{
1 Universidade Federal da Bahia, Escola de Nutrição, Curso de Pós-Graduação em Alimentos, Nutrição e Saúde. R Araújo Pinho, 32, Canela, 40110-150, Salvador, BA, Brasil. Correspondência para/Correspondence to: I.F.B. REZENDE. E-mail: <ionar@superig.com.br>.

2 Universidade Federal da Bahia, Escola de Nutrição, Residência em Nutrição Clínica. Salvador, BA, Brasil.

${ }^{3}$ Universidade Federal da Bahia, Escola de Nutrição, Departamento de Ciências da Nutrição. Salvador, BA, Brasil.
} 
604 | I.F.B. REZENDE et al.

métodos objetivos foi baixo ( $\leq 51,0 \%)$, exceto para albumina e músculo gastrocnêmio (83,0\%). Encontrou-se, para o sexo masculino, Coeficiente de Kendall com valores entre 60,0\% e 70,0\%, representando boa concordância ( $\geq 60,0 \%$ e $<80,0 \%$ ), ao comparar o índice de massa corporal e a circunferência do braço com os músculos da deambulação, da mastigação e dos interósseos.

\section{Conclusão}

1. A Avaliação Muscular Subjetiva apresentou boa reprodutibilidade diagnóstica; 2 . Alterações tróficas subjetivas foram prevalentes nos músculo temporal e masseter; 3. Não foi encontrada concordância excelente entre a Avaliação Muscular Subjetiva e os métodos objetivos de avaliação nutricional para ambos os sexos, exceto entre a albumina e o músculo gastrocnêmio para o sexo feminino.

Termos de indexação: antropometria; avaliação nutricional; cuidados pré-operatórios.

\section{A B S T R A C T}

\section{Objective}

This study will compare Subjective Muscle Assessment with objective methods of nutritional assessment in presurgical patients.

\section{Methods}

This is a cross-sectional study to compare nutritional assessment methods involving primary data in presurgical patients. Anthropometric measurements, laboratory tests and Subjective Muscle Assessment were carried out to assess the subjects' nutritional status. Kendall's tau-b coefficient was employed and values equal to or above $80.0 \%$ were considered excellent in terms of reliability.

\section{Results}

The protocol included 168 adult patients of both genders who would undergo major surgery. For Subjective Muscular Assessment, inter and intra-rater reliability was shown to be $77.0 \%$ and $89.0 \%$, respectively. The muscle groups that presented the greatest percentage of trophic changes were the temporal and the masseter for both genders (female $=53.5 \%$; male=82.0). For women, a low reliability rate $(\leq 51.0 \%$ ) was found between Subjective Muscle Assessment and the objective methods, except for albumin and the gastrocnemius muscle (83.0\%). Kendall's coefficient for men was shown to be $60.0 \%$ and $70.0 \%$, thus revealing good reliability $(\geq 60.0 \%$ and $<80.0 \%$ ) when the body mass index and the arm circumference were compared with walking, mastication and interosseous muscles.

\section{Conclusion}

1. Subjective Muscle Assessment showed good diagnostic reproducibility. 2. Subjective trophic changes prevailed in the temporal and masseter muscles. 3. No excellent reliability was found between Subjective Muscle Assessment and the objective methods of nutritional assessment for both genders, except for that between albumin and the gastrocnemius muscle for women.

Indexing terms: anthropometry; nutritional assessment; preoperative care.

\section{N T R O D U Ç Ã O}

O interesse na avaliação do estado nutricional do paciente hospitalizado tem aumentado nos últimos 20 anos, devido à constatação de grande prevalência de desnutrição, que varia entre $30 \%$ a $50 \%$ nessa população ${ }^{1,2}$.

A desnutrição é um achado comum nos pacientes que serão submetidos a intervenções cirúrgicas, especialmente naqueles com doenças gastrintestinais, mais vulneráveis a condições clínicas como ingestão alimentar insuficiente, má-absorção intestinal e expressiva perda de massa muscular ${ }^{3,4}$.

A assistência nutricional adequada ao paciente submetido a procedimentos cirúrgicos ou a outros tratamentos indutores de imunossupressão favorece a evolução positiva do quadro clínico, a redução da internação hospitalar e a redução dos riscos de complicações ${ }^{5-8}$.

Atualmente a avaliação e a terapia nutricional merecem destaque na área da saúde e 
pesquisa clínica, na qual o nutricionista e demais membros da equipe devem estar capacitados para utilizar corretamente todas as ferramentas disponíveis para um diagnóstico nutricional mais acurado e preciso.

Vários métodos de avaliação nutricional (AN) têm sido propostos, utilizando testes de avaliação clínica, bioquímica, antropométrica e exames de composição corporal. Infelizmente, os critérios diagnósticos possuem limitações importantes. O edema, a falta de padrões de referência para populações brasileiras, os extremos de idade e peso, a doença aguda, a atividade do paciente, o custo elevado e a indisponibilidade de equipamentos apropriados, na maioria dos hospitais, são alguns exemplos dos fatores que dificultam a avaliação nutricional9-12.

A utilização da semiologia torna-se fundamental para o completo diagnóstico nutricional de pacientes hospitalizados. Trata-se da introdução de exame físico criterioso na busca de sinais clínicos de deficiências nutricionais específicas ou atrofia de tecido celular subcutâneo ${ }^{13}$. Os métodos subjetivos são práticos, simples, não-invasivos e sem custo adicional e, quando associados aos parâmetros objetivos (antropométricos e laboratoriais), melhoram a precisão e a acurácia do diagnóstico das desordens nutricionais ${ }^{14,15}$.

A Avaliação Muscular Subjetiva (AMS) é um exame clínico que considera as alterações em quatro grupamentos musculares envolvidos nas atividades rotineiras diárias, tais como a mastigação (temporal/masseter=MTM), a deambulação (gastrocnêmio=MG) e a vida laborativa (adutor do polegar $=M A P / i n t e r o ́ s s e o s=M I)^{16}$. Quando em associação com a história clínica, é capaz de fornecer informações complementares para avaliar o estado nutricional do paciente hospitalizado.

Encontram-se, na literatura científica, dados referentes à prevalência das alterações morfológicas musculares seletivas, relacionadas como método complementar de avaliação nutricional. No entanto, ainda não existem publicações avaliando a AMS de modo comparativo com parâmetros clássicos de avaliação nutricional ${ }^{16}$.
Assim, este estudo pretende trazer uma contribuição sobre o tema, ao avaliar os níveis de concordância da Avaliação Muscular Subjetiva com métodos objetivos de avaliação nutricional em indivíduos que serão submetidos a cirurgias de médio e grande porte em dois hospitais da rede pública.

\section{M É T O D O S}

Foram incluídos no protocolo 168 pacientes adultos, de ambos os sexos, com indicação de cirurgia de médio e grande porte, internados consecutivamente em enfermarias cirúrgicas de dois Hospitais Públicos em Salvador - Bahia, com características similares, durante as primeiras 72 horas de admissão hospitalar.

Os critérios de exclusão adotados foram: pacientes alimentados através de cateteres ou ostomias, exclusivamente, em intervalo superior a um mês; portadores de doenças degenerativas neuromusculares (esclerodermia, neuropatias periféricas), hepáticas, obesidade, síndrome da imunodeficiência adquirida (SIDA) em fase terminal e pacientes com edema que pudesse interferir na realização da avaliação nutricional.

Utilizou-se protocolo de pesquisa detalhado, contendo questões referentes à capacidade funcional, à atividade laborativa e aos hábitos alimentares do paciente.

A antropometria foi realizada por meio de métodos convencionais ${ }^{17,18}$, com auxílio de adipômetro (Langer ${ }^{\circledR}$ ), antropômetro (Rigor e Técnica ${ }^{\circledR}$ ), balança plataforma (Welmy ${ }^{\circledR}$ ) e fita inelástica $\left(\mathrm{Grafco}^{\circledR}\right)$. Foram analisados: peso, altura, Prega Cutânea Tricipital (PCT), Circunferência Braquial (CB), utilizando-se fórmulas matemáticas para o cálculo da Circunferência Muscular do Braço (CMB), da Área Muscular do Braço corrigida $(A M B C)$ e do Índice de Massa Corporal (IMC) $)^{19}$. O cálculo do IMC possibilitou a classificação do estado nutricional de acordo com a Organização Mundial da Saúde ${ }^{20}$. 
Os resultados obtidos para tais indicadores foram relacionados aos valores-padrão do NHANES I (National Health and Nutrition Examination Survey) demonstrados em tabelas de percentis de Frisancho ${ }^{21}$ e classificados, conforme Blackburn \& Thornton ${ }^{22}$, em bom estado nutricional, desnutrição leve, moderada ou grave.

As aferições foram desenvolvidas por duas equipes independentes de antropometristas, por três vezes consecutivas, e sua leitura aritmética correspondeu à média final.

A AMS foi aplicada no mesmo dia em que foi realizada a antropometria, por duas examinadoras independentes, utilizando o protocolo orientado para detectar alterações nos grupamentos musculares específicos: masseter, temporal, adutor do polegar, interróseos e gastrocnêmio. Os critérios utilizados para classificação foram preconizados por Lameu et al. ${ }^{16}$, considerando as alterações encontradas como atrofia leve, moderada, grave ou simplesmente a presença ou ausência de atrofia.

Realizou-se a padronização da coleta dos dados da AMS e das medidas antropométricas, a partir do cálculo da precisão e exatidão intra e intermedidor para aumentar a margem de acertos.

Os parâmetros laboratoriais avaliados foram: albumina sérica, hemoglobina, hematócrito e Contagem Total de Linfócitos (CTL), por técnicas descritas na literatura ${ }^{23,24}$. Os resultados encontrados foram categorizados de acordo com valores de referência adotados por Blackburn \& Thornton ${ }^{22}$ e Sauberlich et al. ${ }^{25}$.

Previamente à coleta dos dados, os pacientes foram informados sobre o objetivo do estudo e assinaram o termo de consentimento livre e esclarecido. O protocolo de pesquisa foi analisado e aprovado pelo Comitê de Ética em Pesquisa da Maternidade Climério de Oliveira da Universidade Federal da Bahia - CEP/CMO/UFBA, parecer No57/2005.

As análises estatísticas foram realizadas utilizando o pacote Stata Corporation 2005/Stata Statistical Software ${ }^{26}$. Realizou-se análise descritiva para as variáveis demográficas e clínicas do estudo. A concordância da AMS inter e intra-observadores foi avaliada por meio do Coeficiente $\mathrm{Kappa}^{27}$. Considerou-se como boa concordância, valores de Kappa acima de 60\%, baixa $\leq$ a $40 \%$ e ótima > que $80 \%$.

Entre os métodos do estudo a medida de ocorrência epidemiológica utilizada para o diagnóstico nutricional foi o Coeficiente de Tau-b de Kendall ${ }^{28}$. Considerou-se como bom grau de concordância quando o coeficiente de Kendall encontrava-se $\geq 60 \%$ e $<80 \%$, baixo $<60 \%$ e excelente $\geq 80 \%$.

\section{RESULTADOS}

Os pacientes que integraram a amostra deste estudo foram, em sua maioria, do sexo feminino $(76,8 \%)$, sendo apenas $23,2 \%$ do sexo masculino. A faixa etária dos indivíduos estudados foi de 20 a 59 anos (Média - M=41,5; Desvio-padrão - DP=11). Os principais diagnósticos clínicos que resultaram na necessidade da intervenção cirúrgica foram: colelitíase, mioma uterino, megaesôfago chagásico, neoplasia de tireóide, de esôfago, estômago, duodeno e cólon.

Os procedimentos cirúrgicos de médio e grande porte foram: Colecistectomia $(35,71 \%)$, Miomectomia (16,67\%), Tireodectomia (13,10\%), Esofagectomia (6,55\%) e outros como Laparotomia Exploradora, Gastrectomia, Colectomia, Esplenectomia, Duodenopancreatectomia, Derivação Biliodigestiva (27,97\%).

Neste estudo, a concordância inter-examinadores foi superior a $77,00 \%(p<0,001)$, refletindo uma boa reprodutibilidade diagnóstica da AMS, entre os investigadores nos dois momentos de avaliação no processo de padronização. A concordância da AMS intra-observadores foi considerada ótima, com valores de Kappa iguais ou superiores a $89,53 \%(p<0,001)$, para as variáveis estudadas: MTM, MAP, MI e MG.

Verificou-se, entre os pacientes do sexo feminino e masculino, que $53,48 \%$ e $82,06 \%$ 
apresentaram alterações no trofismo do músculo temporal e masseter, respectivamente; para o músculo adutor do polegar, as alterações tróficas foram encontradas em $14,73 \%$ das mulheres e em $25,64 \%$ dos homens estudados. Em relação aos músculos interósseos e gastrocnêmio, as alterações foram encontradas, respectivamente, em $17,83 \%$ e em $8,53 \%$ das mulheres; para o sexo masculino, as alterações nesses grupamentos musculares observadas foram similares $(25,64 \%)$. Dessa forma, o grupamento muscular que apresentou o maior percentual de alteração foi o da mastigação $(p<0,05)$, com destaque para os homens do estudo.

Considerando que foi definido como excelente concordância, entre os métodos objetivos e o subjetivo, coeficiente Tau-b de Kendall igual ou superior a $80,00 \%$, observou-se que o grau de concordância entre a AMS e os métodos objetivos de avaliação nutricional, para as mulheres do estudo, mostrou-se, de um modo geral, baixo, uma vez que o maior coeficiente de concordância foi igual ou inferior a 51,00\% (Figuras 1 a 4), com exceção do valor excelente de concordância (superior a 80,00\%) entre o músculo gastrocnêmio e a albumina (83,12\%). Entre os parâmetros

Grau de concordância AMS X antropometria

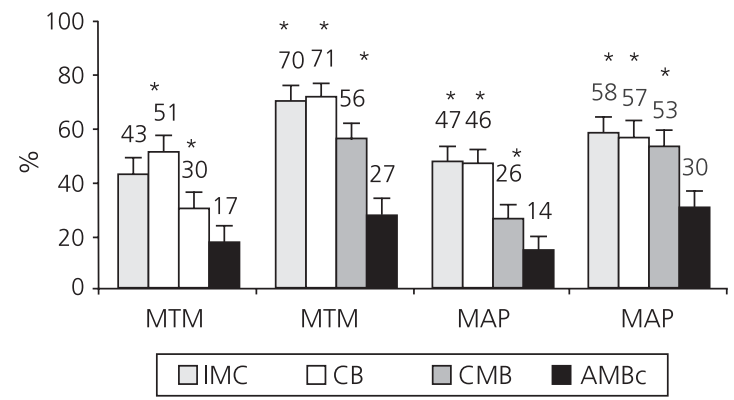

Figura 1. Grau de concordância entre avaliação muscular subjetiva (MTM/MAP) e antropometria dos pacientes de ambos os sexos no pré-operatório. Salvador, BA, 2005.

MTM: músculo temporal e masseter; MAP: músculo adutor do polegar; IMC: índice de massa corporal; CB: circunferência do braço; CMB: circunferência muscular do braço; AMBc: área muscular do braço corrigida.

${ }^{*} p$ valor $<0,05$ (entre grupamentos musculares e indicadores antropométricos por sexo). objetivos, a AMBc e a Contagem Total de Linfócitos foram os indicadores que apresentaram maior discordância entre si.

Grau de concordância AMS X antropometria

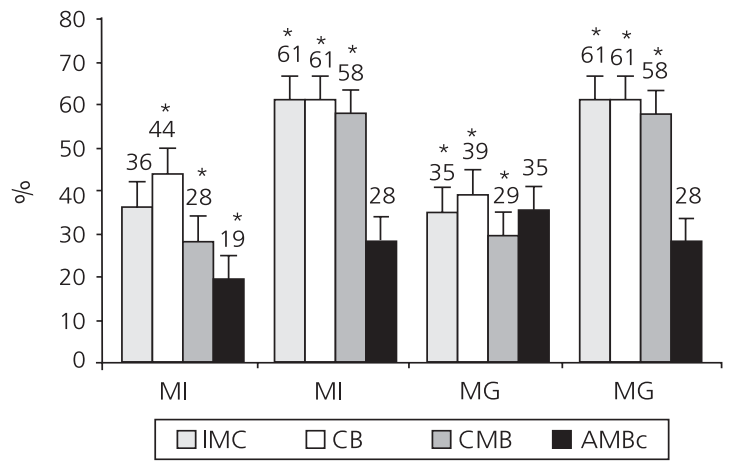

Figura 2. Grau de concordância entre avaliação muscular subjetiva (MI/MG) e antropometria dos pacientes de ambos os sexos no pré-operatório. Salvador, BA, 2005.

MI: músculos interósseos; MG: músculo gastrocnêmio; IMC: índice de massa corporal; $C B$ : circunferência do braço; $C M B$ : circunferência muscular do braço; $\mathrm{AMBc}$ : área muscular do braço corrigida.

${ }^{*} p$ valor $<0,05$ (entre grupamentos musculares e indicadores antropométricos por sexo).

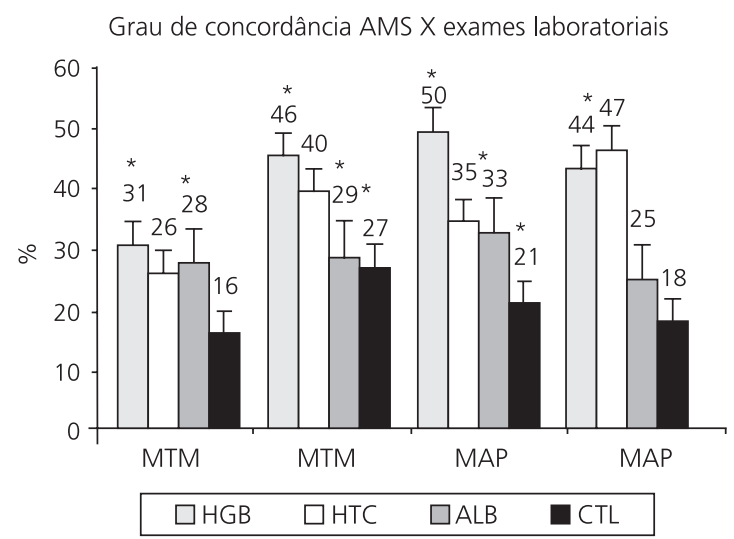

Figura 3. Grau de concordância entre a avaliação muscular subjetiva (MTM/MAP) e exames laboratoriais dos pacientes de ambos os sexos no pré-operatório. Salvador, BA, 2005.

MTM: músculo temporal e masseter; MAP: músculo adutor do polegar; HGB: hemoglobina; HTC: hematócrito; ALB: albumina; CTL: contagem total de linfócitos.

${ }^{*} p$ valor $<0,05$ (entre grupamentos musculares e indicadores laboratoriais por sexo). 


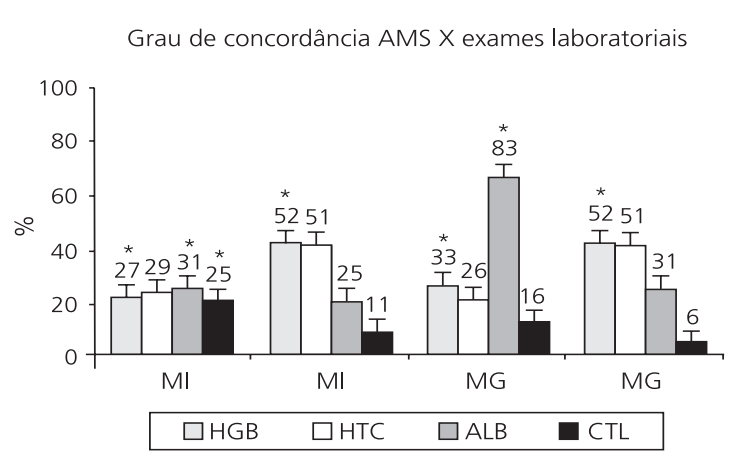

Figura 4. Grau de concordância entre avaliação muscular subjetiva (MI/MG) e exames laboratoriais dos pacientes de ambos os sexos no pré-operatório. Salvador, BA, 2005.

MI: músculos interósseos; MG: músculo gastrocnêmio; HGB: hemoglobina; HTC: hematócrito; ALB: albumina; CTL: contagem total de linfócitos.

${ }^{*} p$ valor $<0,05$ (entre grupamentos musculares e indicadores laboratoriais por sexo).

Em relação aos métodos antropométricos e alguns indicadores da AMS, encontrou-se, para os homens do estudo, boa concordância (IMC; CB × MTM; MI; MG), com exceção da CMB e $A M B c$, mas não foi encontrado nenhum indicador com excelente concordância. Para os métodos laboratoriais houve discordância entre todos os indicadores, considerando que o maior coeficiente de Tau-b de Kendall foi 52,27\%. De modo geral, não houve concordância excelente entre os parâmetros avaliados considerando ambos os sexos (Figuras 1 a 4).

\section{DIS C U S S Ã O}

\section{Reprodutibilidade diagnóstica da AMS}

A mensuração e a observação de cada procedimento que compõe a investigação científica visam a alcançar uma padronização adequada, de modo que as estatísticas resultantes representem a realidade com a precisão desejada. Para tanto, a confiabilidade é um dos instrumentos disponíveis para verificação da qualidade do método aplicado ${ }^{27}$.
Por se tratar de método subjetivo, a AMS tem sua precisão diagnóstica dependente do observador. Nesse sentido, nosso estudo apresentou consistência dos resultados, quando a AMS foi repetida, com concordâncias inter e intra-examinadores superiores a $77,0 \%$ e $89,5 \%$, refletindo, portanto, uma boa e ótima reprodutibilidade, respectivamente. Esses dados são similares aos achados na literatura, na qual Lameu et al. ${ }^{16}$ demonstraram, em pacientes clínicos hospitalizados, 90\% de concordância relacionada à AMS.

Dessa forma, os resultados da sistematização da coleta de dados por meio da AMS, com passos definidos e ordenados de conduta, tendem a melhorar a qualidade da informação e, conseqüentemente, a concordância entre profissionais de saúde que usam tal procedimento.

\section{Avaliação muscular subjetiva}

Na presente investigação científica avaliou-se a prevalência de atrofia muscular subjetiva em 168 pacientes masculinos e femininos no período pré-operatório, sendo possível detectar alterações morfológicas nos músculos responsáveis pela mastigação, pela vida laborativa e pela deambulação, consistentes com os achados obtidos por Lameu et al. ${ }^{16}$, em 89 pacientes clínicos hospitalizados. Assim, demonstrou-se a ocorrência de um percentual considerável de indivíduos hospitalizados com atrofia no músculo da mastigação. Para os demais grupamentos musculares (interósseos, adutor e gastrocnêmio) observou-se atrofia, porém não tão elevada, refletindo a perda de massa magra, a perda de vida laborativa e a redução da deambulação, respectivamente. Essas informações são importantes para complementar o resultado da avaliação nutricional na população estudada.

Os resultados da avaliação muscular subjetiva nesses pacientes pré-operatório estão consoantes com dados da literatura, confirmando que o trofismo da massa esquelética é mantido pela atividade física ${ }^{29}$, pela inervação ${ }^{30}$, pela irrigação ${ }^{31}$ 
e pela oferta nutricional ${ }^{32}$, sendo que qualquer alteração em um desses fatores pode ocasionar atrofia muscular.

Nesta pesquisa, observou-se, entre os pacientes com atrofia leve e moderada dos músculos temporal e masseter, a presença de hábitos de mastigação reduzida, modificação da consistência da dieta e sintomas gastrintestinais, como disfagia, náusea, vômitos e anorexia, condizentes com outros relatos da literatura ${ }^{3,33}$. É importante salientar que esses fatores predisponentes podem contribuir para o aparecimento de alterações morfológicas nesses grupamentos musculares com maior exposição do arco zigomático correlacionando-se com a redução de reserva energética. Entretanto, para que haja relação entre a atrofia dos músculos da mastigação e a desnutrição, é necessário que as alterações musculares estejam presentes em grau avançado (sinal de chave), significando perda protéico-energética prolongada.

No que diz respeito ao MAP, os resultados com pacientes cirúrgicos no pré-operatório reproduziram o princípio descrito por Gea et al. ${ }^{34}$ e Man et al..$^{35}$, segundo o qual, apesar da gravidade da doença básica, a massa e a função do adutor do polegar são mantidas enquanto é possível o paciente preservar a atividade laborativa. A apatia, comum na desnutrição, ocasiona redução na força e na taxa de relaxamento muscular, com conseqüente aumento da fadiga muscular ${ }^{36}$.

Entretanto, em pequena parte da amostra, observou-se perda da massa muscular do adutor do polegar, em graus variados de atrofia, de leve $(21,11 \%)$ a moderada $(19,26 \%)$, sugerindo redução recente da vida laborativa e piora do quadro funcional. Observou-se que a atrofia muscular desse grupamento estava relacionada, principalmente, ao desuso progressivo do músculo adutor do polegar, nos pacientes submetidos a cirurgia de grande porte do trato digestório, cursando com limitações na ingestão alimentar, decorrentes da doença de base que, indiretamente, conduziram à desnutrição e à letargia12,37.

Em relação à musculatura interóssea das mãos, encontrou-se atrofia nesse grupamento para pacientes no pré-operatório com valores similares aos encontrados por Lameu et al. ${ }^{16}$, em pacientes clínicos. Esses achados refletem a perda de massa magra, sugerindo redução na ingestão alimentar em indivíduos hospitalizados ${ }^{38}$. As alterações nos músculos interósseos foram detectadas naqueles pacientes que, provavelmente, apresentaram algum sintoma gastrintestinal, secundário à doença de base, conseqüentemente, comprometendo a ingestão de nutrientes energéticos e plásticos.

O grupamento muscular responsável pelo menor percentual de atrofia foi o gastrocnêmico (feminino=8,53\%; masculino=25,64\%), sugerindo que, nesta população de pacientes pré-cirúrgicos, a capacidade de deambulação estava, na maioria das vezes, preservada.

\section{Concordância diagnóstica entre métodos}

Os resultados deste estudo indicam que o grau de concordância entre a AMS (MTM, MAP, $M I, M G$ ) e os métodos antropométricos (IMC, CB, $\mathrm{CMB}, \mathrm{AMBC}$ ) apresentou-se comumente baixo para avaliação do estado nutricional dos pacientes cirúrgicos pré-operatório do sexo feminino. Para essa comparação utilizaram-se, como ponto de corte de concordância entre esses indicadores, valores superiores ou iguais a $80 \%$ do coeficiente de Tau-b de Kendall ${ }^{39}$.

Vale ressaltar que não existem relatos na literatura utilizando a AMS no período pré-operatório, de modo comparativo com parâmetros clássicos de avaliação nutricional. Assim, discutiu-se o tema de forma a estimar o grau de concordância entre os métodos, relacionando-o com o sexo.

Observou-se fraca concordância entre a AMS e os indicadores bioquímicos (albumina, CTL, hemoglobina e hematócrito), para o sexo feminino, com exceção do coeficiente encontrado com excelente concordância (coeficiente Tau-b de Kendall $=83,12 \%$ ) entre o MG e a albumina. A excelente concordância encontrada entre os dois métodos não foi observada em relação aos outros 
parâmetros utilizados para a avaliação nutricional Dessa forma, sugere-se a realização de outros trabalhos que investiguem os níveis de reprodutibilidade sobre as características dos indicadores observados.

Verificou-se que os métodos investigados não apresentaram concordância significativa na identificação de pacientes com estado nutricional depletado, entre os indivíduos do sexo feminino.

De acordo com a análise processada para avaliar o grau de concordância entre os métodos em discussão, encontrou-se, para o sexo masculino, coeficiente de Kendall com valores entre $60 \%$ e $70 \%$, representando boa concordância ( $\geq 60 \%$ e $<80 \%$ ) ao comparar o IMC e a CB com MTM, Ml e MG. No entanto, não foi demonstrada excelente concordância (coeficiente de Tau-b de Kendal $\geq 80 \%$ ) entre a AMS, os métodos antropométricos e os exames laboratoriais, com exceção da albumina e MG.

A avaliação os resultados a partir dos grupamentos musculares citados, permite inferir que os músculos da mastigação, da deambulação e os músculos interróseos apresentaram boa concordância, tanto com o IMC quanto com a CB. Considerando que definiu-se como ponto de corte para concordância excelente entre os métodos valores superiores ou iguais a $80 \%{ }^{39}$, provavelmente, para o sexo masculino a AMS não refletiu associação de forma fidedigna com o estado nutricional comprometido.

No entanto, observou-se que a proporção de concordância registrada entre os indicadores avaliados, para ambos os sexos, não foi excelente, apresentando baixo poder discriminatório na avaliação das alterações nutricionais dos pacientes estudados.

Pode-se observar, ainda, na presente pesquisa, que o exame subjetivo do músculo adutor do polegar apresentou os menores valores de concordância com os métodos objetivos, provavelmente, devido às peculiaridades desse grupamento muscular, entre as quais a perda subjetiva do adutor pode estar relacionada com a redução da vida laborativa induzida pela doença. Além disso, a manutenção do trofismo do adutor do polegar pode ocorrer independentemente da perda de massa global muscular 34,35

Similarmente a achados da literatura específica, estes resultados mostraram que o exame morfológico muscular só permite avaliar a desnutrição após um período variável de tempo (cerca de dois a quatro meses), limitando sua utilização para diagnosticar as formas agudas de alterações nutricionais ${ }^{12}$. Em outras palavras, com a utilização dessa técnica, pequenas alterações do estado nutricional ao exame físico não seriam facilmente visualizadas.

Entretanto, é oportuno salientar que os demais métodos objetivos indicados na prática clínica para realização da avaliação nutricional, também não possuem precisão suficiente para detectar as mínimas variações na composição corporal ocorridas em pequenos períodos de tempo.

Assim, o baixo poder de concordância encontrado nos resultados desta pesquisa, entre os indicadores mencionados para avaliar o estado nutricional pré-operatório dos pacientes hospitalizados, possivelmente, seja o reflexo da falta de um conceito universal para definir a desnutrição. Nesse sentido, ao considerar a definição de desnutrição um tanto complexa, é natural que a tarefa de mensurar tal condição também possua alto grau de complexidade, considerando as inúmeras possibilidades para avaliação nutricional, sendo que não existe um único indicador passível de uso em todas as situações clínicas, com a mesma acurácia.

Dessa forma, salienta-se que as diferentes situações clínicas requerem diferentes métodos, embora muitos tendam a se relacionar estritamente entre si. Além do exposto anteriormente, é interessante destacar que a seleção dos indicadores mais apropriados também depende dos objetivos da questão científica formulada.

Diante dos resultados, e tomando por base a literatura consultada, pode-se enfatizar que 
ainda não está disponível um indicador que, isoladamente, seja capaz de mensurar a desnutrição em pacientes cirúrgicos e que consiga abranger todas as especificidades das situações clínicas.

Contudo, a fraca concordância registrada neste estudo, entre os métodos objetivos e a mas, não deve interferir na definição de prioridades com respeito à determinação de parâmetros que, associados, possam refletir o estado nutricional da população estudada de forma mais confiável.

Nesse sentido, a AMS tem se mostrado um achado útil para complementar a avaliação e o acompanhamento clínico da assistência nutricional a pacientes hospitalizados, por sua capacidade de detectar alterações morfológicas na musculatura responsável pela mastigação, deambulação e vida laborativa. O presente estudo reforça a importância desse método enquanto parte da avaliação nutricional do paciente hospitalizado, principalmente, naqueles pacientes que serão submetidos a procedimentos cirúrgicos de médio e grande porte.

Apesar de possibilitar a participação de qualquer profissional de saúde na realização da AMS, é indispensável o treinamento adequado de todos os observadores que desejam praticá-la, pois a precisão depende da experiência do examinador.

Sugere-se a complementação da AMS com outras técnicas de avaliação nutricional, bem como a utilização ou criação de índices múltiplos, com o objetivo de aumentar a sensibilidade e a especificidade no diagnóstico das alterações nutricionais, uma vez que, até o momento, não existe parâmetro tradicional isolado capaz de estabelecer diagnóstico nutricional mais fidedigno para o paciente hospitalizado.

Recomenda-se a realização de mais estudos sobre este método, nos quais poderão ser testados outros protocolos da AMS e selecionada amostra com pacientes desnutridos para avaliação de complicações no pós-operatório.

\section{O N C L U S Ã O}

Nas condições da presente pesquisa, em que pacientes avaliados no pré-operatório de cirurgias de médio e grande porte foram submetidos à avaliação nutricional, por métodos objetivos e Avaliação Muscular Subjetiva (AMS), pode-se concluir que:

1. A AMS apresentou boa reprodutibilidade diagnóstica inter e intra-examinadores;

2. A prevalência de alterações tróficas subjetivas nos grupamentos musculares estudados ocorreu de forma importante, principalmente, no músculo temporal e masseter;

3. Não foi observada concordância significativa entre a AMS e os métodos objetivos de avaliação nutricional para ambos os sexos, exceto entre a albumina e o MG para o sexo feminino.

\section{COLABORADORES}

I.F.B. REZENDE, mentora do trabalho cientifico, responsável pelo planejamento e execução do protocolo de pesquisa, planejamento, execução e a supervisão do projeto de pesquisa em todas as etapas, bem como a elaboração do artigo. R.P.J. MAZZA, planejamento do protocolo do projeto, elaboração do artigo e planejamento do projeto como orientadora. L.R. SAMPAIO colaborou na análise dos dados e na elaboração do artigo. A.S. ARAÚJO colaborou na coleta dos dados, realização da avaliação antropometrica. M.F. SANTOS colaborou na coleta dos dados, realização da avaliação antropométrica.

\section{A GRADECIMENTO}

Ao Dr. Edson Lameu pelos esclarecimentos prestados.

\section{REFER Ê N CIAS}

1. Waitzberg DL, Caieffe WT, Correia MITD. Inquérito brasileiro de avaliação nutricional hospitalar (Ibranutri). Rev Bras Nutr Clin. 1999; 14:124-34. 
2. Barbosa-Silva Barros JO. Avaliação nutricional subjetiva. Parte 1 - Revisão de sua validade após duas décadas de uso. Arq Gastroenterol. 2002; 39(3):181-7.

3. Sena FG, Taddeo EF, Andrade Neto ER, Ferreira MCR, Rolim EG. Estado nutricional de pacientes internados em enfermaria de gastroenterologia. Rev Nutr. 1999; 12(3):233-9.

4. Correia MITD, Caiaffa WT, Silva AL, Waitzberg DL. Risk factors for malnutrition in patients undergoing gastroenterological and hérnia surgery: an analysis of 374 patients. Nutr Hosp. 2001; 16(2):59-64.

5. Coast KG, Morgan SL, Bartolucci AA, Weinsier RL. Hospital-associated malnutrition: a reevaluation 12 years later. J Am Diet Assoc. 1993; 93(1):27-33.

6. McWhirter JP, Hill K, Pennington CR. The nutritional status of patients with gastrointestinal disease. Nutrition. 1994; 10:495.

7. Incalzi RA, Gemma A, Capparella O, Cipriani L, Landi F, Carbonin P. Energy intake and in-hospital starvation. A clinically relevant relationship. Arch Inter Med. 1996; 156(4):425-9.

8. Corish C, Flood P, Mulligan S, Kennedy NP. Prevalence of undernutrition and weight loss changes during the course of hospitalization among patients admitted to two Dublin hospitals. Proceed Nutr Soc. 1998; 57:10.

9. Mullen JL, Buzby GP, Waldman MT, Gertner MH, Hobbs CL, Rosato EF. Prediction of operative operative morbidity and mortality by preoperative nutritional assessment. Surg Forum. 1979; 30: 80-2.

10. Grant JP. Nutritional assessment by body compartment analysis. In: Grant JP, editor. Handbook of total parenteral nutrition. New York: WB Saunders; 1992. p.15.

11. Manning EM, Shenin A. Nutritional assessment in the critically ill. Crit Care Clin. 1995; 11(3):603-34.

12. Lameu E, Andrade PV-B, Luiz RR. Avaliação muscular subjetiva. In: Lameu E, editor. Clínica nutricional. Rio de Janeiro: Revinter; 2005. p.179-87.

13. Duarte CD, Castellani FR., editores. Semiologia nutricional. Rio de Janeiro: Axcel Books; 2002.

14. Klein S, Kinney J, Jeejeebhoy KN, Alpes D, Hellerstein M, Murray $M$, et al. Nutrition support in clinical practice: a review of published data and recommendations for future research directions. J P N J Parenter Enteral Nutr. 1997; 21(3):133-56.

15. Corish CA, Kennedy NP. Protein-energy undernutrition in hospital in-patients. $\mathrm{Br} J$ Nutr. 2000; 83(8):575-91.
16. Lameu EB, Andrade P, Gerude M. Avaliação muscular específica e subjetiva em pacientes clínicos. Rev Bras Nutr Clin. 2003; 18(Supl):S24.

17. Lohman TG, Roche AF, Martorell R, editores. Anthropometric standardization reference manual. New York: Champaign Human Kinetics Books; 1988.

18. Lohman TG, Roche AF, Martorell R. Anthropometric standardization reference manual. Abridged edtion; 1991.

19. Heymsfield SB, McMannus CB, Smith J. Anthropometric measurement of muscle. Revised equations for calculating bone-free arm muscle area. Am J Clin Nutr. 1982; 36(4):680.

20. World Health Organizarion. Physical status: the use and interpretation of anthropometry. Geneva; 1995. WHO Technical Report Series, n. 854.

21. Frisancho AR. Anthropometric standards for the assessment of growth and nutritional status. Ann Arbor, Michigan: University of Michigan Press; 1990.

22. Blackburn GL, Thornton PA. Nutritional assessment of the hospitalized patients. Med Clin North Am. 1979; 63(5):1103-15.

23. Kingsley GR. The direct biuret method for the determination of serum priteins as applied to photoelectric and visual colorimetry. J Lab Clin. 1942; 27:840-5.

24. Henhy RJ, Sobel C, Berkman S. Interferences with biuret methods for serum proteins. Anal Chem. 1957; 29:1491-5.

25. Sauberlich HE, Dowdy RP, Skala JH. Laboratory tests for the assessment of nutritional status. CRC Crit Rev Clin Lab Sci. 1973; 4(3):215-340.

26. Stata Corporation Stata. Statistical Software. Release 9. College Station, Texas: Stata Corporation LP; 2005.

27. Pereira MG. Epidemiologia teoria e prática. Rio de Janeiro: Guanabara Koogan; 1995.

28. Darroch J, McCloud PI. Category of distinguishability and observer agreement. Aust J Stat. 1986; 28:371-88.

29. Patel AN, Razzak ZA, Dastur DK. Disuse atrophy of human skeletal muscle. Arch Neurol. 1969; 20(4):413.

30. Cruz-Martinez A, Arpa J. Reinnervation of dystrophic muscles. Acta Neurol Scand. 2001; 104(4):236-38.

31. Wright JR, McLoskey DI, Fitzpatrick RC. Effects of muscle perfusion pressure on fatigue and systemic arterial pressure in human subjects. J Appl Physical, 1999; 86(3):845. 
32. Russell DM, Walker PM, Leite LA. Metabolic and structural changes in skelectal muscle during hypocaloric dieting. Am J Clin Nutr. 1984; 39(4): 503-13.

33. Rezende IFB, Oliveira VS, Kuwano EA, Leite APB, Rios I, Dórea YSS, et al. Prevalência da desnutrição hospitalar em pacientes internados em um hospital filantrópico em Salvador, Bahia, Brasil. Rev Cien Med Biol. 2004; 3(2):194-200.

34. Gea J, Orozco-Levi M, Barreiro E, Ferrer A, Broquetas J. Structural and functional changes in the skeletal muscles of COPD patientes: the "compartments" theory. Monaldi Arch Chest Dis. 2001; 56(3):214-24.

35. Man WDC, Soliman MGG, Nikoletou D, Harris ML, Rafferty GF, Mustfa MI, et al. Non-volitional assessment of skeletal strength in patients with chronic obstructive pulmonary disease. Thorax. 2003; 58(8):665-9.
36. Lopes J, Russel DM, Whitwell J, Jeejeebhoy KN. Skeletal muscle function in malnutrition. Am J Clin Nutr. 1982; 36(4):602-10.

37. Waitzberg DL, Terra RM. Função muscular e sua relação com nutrição e desnutrição. In: Waitzeberg $\mathrm{DL}$, editor. Nutrição enteral e parenteral na prática clínica. 2a. ed. São Paulo: Atheneu; 2000. p.321-5.

38. Gerud M. Musculatura adutora do polegar: um novo parâmetro antropométrico [dissertação]. Rio de Janeiro: Universidade Federal do Rio de Janeiro; 2003.

39. Fleiss JL. Stastistical methods for rates and proportion. New York: Wiley; 1981.

Recebido em: 9/2/2007

Versão final reapresentada em: 20/7/2007

Aprovado em: 10/10/2007 